\title{
Nonrealizability Proofs in Computational Geometry
}

\author{
Jürgen Bokowski, ${ }^{1}$ Jürgen Richter, ${ }^{1}$ and Bernd Sturmfels ${ }^{2}$ \\ 'Fachbereich Mathematik, Technische Hochschule Darmstadt, Schlossgartenstrasse 7, \\ D-6100 Darmstadt, Federal Republic of Germany \\ ${ }^{2}$ Research Institute for Symbolic Computation, Johanes-Keplet Universität Linz, \\ A-4040 Linz, Austria
}

\begin{abstract}
This paper deals with a class of computational problems in real algebraic geometry. We introduce the concept of final polynomials as a systematic approach to prove nonrealizability for oriented matroids and combinatorial geometries.

Hilbert's Nullstellensatz and its real analogue imply that an abstract geometric object is either realizable or it admits a final polynomial. This duality has first been applied by Bokowski in the study of convex polytopes [7] and [11], but in these papers the resulting final polynomials were given without their derivations.

It is the objective of the present paper to fill that gap and to describe an algorithm for constructing final polynomials for a large class of nonrealizable chirotopes. We resolve a problem posed in [10] by proving that not every realizable simplicial chirotope admits a solvability sequence. This result shows that there is no easy combinatorial method for proving nonrealizability and thus justifies our final polynomial approach.
\end{abstract}

\section{Introduction}

A large class of realization problems in computational geometry can be reduced to the realizability problem of chirotopes or equivalently of oriented matroids [10]. Among such problems which have been studied in the recent literature are the polytopality of combinatorial spheres [11] and [25], the embeddings of triangulated manifolds [5] and [6] and the stretchability of pseudoline arrangements [20]. Apart from these applications, the realizability problem for both oriented and unoriented matroids is a fundamental theoretical question in both matroid theory [30] and algebraic geometry [16] and [27].

Throughout the literature most nonrealizability proofs for (oriented) matroids and nonpolytopality proofs for spheres were based on arguments from classical 
projective geometry. These arguments are often very specific for the object in question and cannot be generalized. Moreover, it is known that over the real numbers there is no finite excluded minor characterization of the geometrically possible configurations [12] and [25]. For these two reasons, it becomes important to develop general computer algebra techniques for proving or disproving realizability of abstract geometric objects.

It is the objective of this paper to suggest such a technique. The method of final polynomials has already been used by the first author in earlier papers [7] and [11] where only the results of the computations were given and applied to the geometric problem in question. In this paper we aim to give a satisfactory answer to the question of how to find a final polynomial for a given nonrealizable structure.

It follows from the results in [26] that deciding the realizability of arbitrary (oriented) matroids is as difficult as solving arbitrary polynomial equations and inequalities with integer coefficients. From a purely theoretical point of view this settles the problem because there are well-known decision procedures for the theory of real closed fields; we refer in particular to Collins's cylindrical algebraic decomposition method [14] and the very recent work of Grigoryev and Vorobjov [17] and Canny [13].

Computer experiments of B. Kutzler show, however, that all inequality systems resulting from the geometric applications in this paper are much too large for the SAC-II implementation of Collins's method. To the best of our knowledge, this implementation is the state of the art in general purpose real geometry software, and so there is a need to develop more specialized but faster algorithms.

There is another important reason why chirotope realizability could be of interest to computer scientists and real algebraic geometers.

The systems of sharp determinantal inequalities resulting from chirotopes share many structural features with problems arising in concrete applications such as robotics [13]. To take full advantage of these specific features, there should be some hope that the techniques to be presented here can be further developed along with the above methods from computer algebra to yield applicable decision procedures for a large class of interesting and applied problems in geometry.

Throughout this paper the algorithmic point of view will be in the foreground. The sections are organized as follows. In Section 2 we explain some basic ideas by discussing a nonrealizable simplicial 3-chirotope with 10 points which has the structure of the Desargues configuration. We give a final polynomial for that example, and we describe in detail how this final polynomial has been constructed. Earlier Bokowski used precisely this construction technique to solve several previously open mathematical problems concerning the realizability of fourdimensional polytopes [7] and [11].

In Section 3 we begin the discussion of the general case. We give a "geometric preprocessing" algorithm which performs the following inequality reduction. Starting with a very large set of determinantal inequalities, we obtain a substantially smaller inequality system which is still equivalent to the original system. Once this reduced system has been found, the original geometric structure can be 
ignored, and we proceed by variable elimination. If this elimination process leads to a contradiction, e.g., if " $0<0$ " can be derived in a certain sequence of deductive steps from the reduced system, then this sequence can be merged to a single (final) polynomial which shows the contradiction.

In Section 5 we turn to the coordinatization algorithm which has been introduced by Bokowski and Sturmfels in [10]. There it was conjectured that the absence of a solvability sequence is insufficient for the nonrealizability of a chirotope. We prove this conjecture by establishing a realizable simplicial 3chirotope $R_{3}^{9}$ with nine points due to J. Richter which does not admit a solvability sequence. Although $R_{3}^{9}$ does fulfill the isotopy property [10, Section 6], this example suggests the existence of chirotopes with disconnected realization space. Very recently, after the completion of this paper, it has been proved by White [31] and Jaggi and Mani-Levitska [18] that there are chirotopes without the isotopy property.

Finally, we discuss final polynomials from a general algebraic geometry point of view in Section 6 . We outline the proof for the existence of final polynomials for all nonrealizable matroids and oriented matroids. This result was independently obtained by Dress and Sturmfels [27].

The reader is referred to [10], [15], [19], [22], and [27] for the basic concepts of oriented matroid theory. Throughout this paper we identify oriented matroids with their chirotope representation. In order to define chirotopes, we denote the set of ordered $d$-tuples of $n$ elements by

$$
\Lambda(n, d):=\left\{\left(\lambda_{1}, \ldots, \lambda_{d}\right) \mid 1 \leq \lambda_{1}<\lambda_{2}<\cdots<\lambda_{d} \leq n\right\} .
$$

Definition. A mapping $\chi: \Lambda(n, d) \rightarrow\{-1,0,+1\}$ is an (oriented) $d$-chirotope with $n$ vertices if for all $\lambda \in \Lambda(n, d+1)$ and for all $\mu \in \Lambda(n, d-1)$ the set

$$
\left\{(-1)^{i} \cdot \chi\left(\lambda_{1}, \ldots, \hat{\lambda}_{i}, \ldots, \lambda_{d+1}\right) \cdot \chi\left(\mu_{1}, \ldots, \mu_{d-1}, \lambda_{i}\right) \mid i \in\{1, \ldots, d+1\}\right\}
$$

either contains $\{-1,+1\}$ or equals $\{0\}$. Here we identify $\chi$ with its unique alternating extension $\chi:\{1, \ldots, n\}^{d} \rightarrow\{-1,0,+1\}$.

The chirotope $\chi$ is called simplicial if $\chi(\Lambda(n, d)) \subset\{-1,+1\}$, and $\chi$ is realizable if there exist vectors $x_{1}, x_{2}, \ldots, x_{n} \in R^{d}$ such that

$$
\text { sign } \operatorname{det}\left(x_{\lambda_{1}}, \ldots, x_{\lambda_{d}}\right)=\chi\left(\lambda_{1}, \ldots, \lambda_{d}\right) \quad \text { for all } \lambda \in \Lambda(n, d) \text {. }
$$

Using homogeneous coordinates, we identify $(d-1)$-dimensional affine space with a hyperplane in $R^{d}$ which does not contain the origin. With a given configuration of $n$ points $x_{1}, x_{2}, \ldots, x_{n}$ in the affine plane, we thus associate the 3-chirotope $\chi$ which assigns to every triple $(i, j, k)$ the orientation of the triangle $x_{i}, x_{j}, x_{k}$.

\section{Example: A Simplicial Non-Desargues Chirotope}

Consider the affine 3-chirotope $D_{3}^{10}$ associated with the pseudoconfiguration in Fig. 1. Interpreting the curved lines as straight lines, we can read off the orientation 


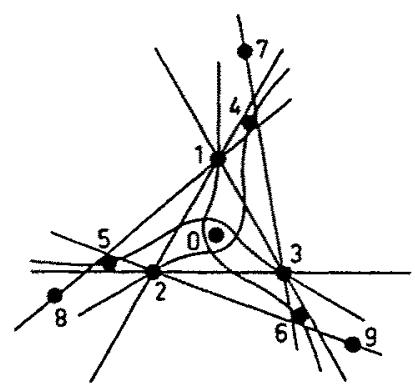

Fig. 1. The nonrealizable chirotope $D_{3}^{10}$.

$[i j k] \pm$ of each triple $(i, j, k) \in \Lambda(10,3)$ of points in $D_{3}^{10}$. For example, we get $[123]+,[124]+,[125]-, \ldots,[145]+,[146]-,[147]+,[148]+, \ldots,[158]-$, etc. It is possible to show that $D_{3}^{10}$ is not realizable by a geometric argument based on Desargues theorem. Here, however, we want to give an algebraic nonrealizability proof by exhibiting a final polynomial for $D_{3}^{10}$. Recall the following well-known identities:

Proposition 2.1 (Grassmann-Plücker Syzygies). Given any field $K$ and $x, y, z \in$ $K^{3}$, we abbreviate the determinant $\operatorname{det}(x, y, z)$ by the bracket $[x y z]$. Then for all $a, b, c, d, e, f \in K^{3}$ we have the identities

$$
\begin{aligned}
&\{a \mid b c d e\}:=[a b c][a d e]-[a b d][a c e]+[a b e][a c d]=0, \\
&\langle a b c \mid \operatorname{def}\rangle:=[a b c][a b c][\operatorname{def}]-\operatorname{det}\left(\begin{array}{lll}
{[d b c]} & {[a d c]} & {[a b d]} \\
{[e b c]} & {[a e c]} & {[a b e]} \\
{[f b c]} & {[a f c]} & {[a b f]}
\end{array}\right)=0 .
\end{aligned}
$$

Nonrealizability Proof for $D_{3}^{10}$. Assume that there exist points $x_{1}, x_{2}, \ldots, x_{10}$ in the real Euclidean plane such that for all $i, j, k$ the oriented area $[i j k]$ of the triangle $x_{i}, x_{j}, x_{k}$ has the sign as prescribed in Fig. 1.

The following expression vanishes by Proposition 2.1:

$$
\begin{aligned}
& \{1 \mid 2345\}[234][153][126][126][230][137][183][293] \\
& -\{2 \mid 1340\}[234][153][153][126][126][137][183][293] \\
& +\{1 \mid 2360\}[234][134][152][152][263][137][183][293] \\
& -\{3 \mid 1246\}[134][152][152][263][120][137][183][293] \\
& -\{2 \mid 1356\}[234][134][152][126][103][137][183][293] \\
& +\{3 \mid 1250\}[234][134][152][126][126][137][183][293] \\
& -\{1 \mid 2358\}[134][263][137][293][120]([234][153][126]+[134][152][263]) \\
& +\{2 \mid 1369\}[234][153][137][183][120]([234][153][126]+[134][152][263]) \\
& -\{3 \mid 1247\}[153][263][183][129][120]([234][153][126]+[134][152][263]) \\
& +\langle 123 \mid 789\rangle[134][153][263][120]([234][153][126]+[134][152][263])
\end{aligned}
$$


By expanding the Grassmann-Plücker terms $\{\cdots \mid \cdots\}$ and $\langle\cdots \mid \cdots\rangle$ in the above polynomial, we obtain after cancellation of several summands

$$
\begin{aligned}
& {[123][145][234][153][126][126][230][137][183][293] } \\
+ & {[123][240][234][153][153][126][126][137][183][293] } \\
+ & {[123][160][234][134][152][152][263][137][183][293] } \\
+ & {[123][364][134][152][152][263][120][137][183][293] } \\
+ & {[123][256][234][134][152][126][103][137][183][293] } \\
+ & {[123][350][234][134][152][126][126][137][183][293] } \\
+ & {[123][296][234][153][137][183][120]([234][153][126]+[134][152][263]) } \\
+ & {[123][374][153][263][183][129][120]([234][153][126]+[134][152][263]) } \\
+ & {[123][185][134][263][137][293][120]([234][153][126]+[134][152][263]) } \\
+ & ([123][289]+[172][183][293]+[172][283][139]+[182][237][139] \\
& +[129][137][283]) \\
& {[134][153][263][120]([234][153][126]+[134][152][263]) }
\end{aligned}
$$

All brackets occurring in this polynomial, e.g., [123], [145], . , [374], [153],.., have to be positive in any realization of $D_{3}^{\text {to }}$ as is seen from Fig. 1. This is a contradiction because the sum of 10 positive numbers cannot vanish. This completes the nonrealizability proof of $D_{3}^{10}$.

Such a polynomial which "obviously" shows the nonrealizability of a chirotope is called a final polynomial, a notion that is more precisely defined in Section 6. In this section our main goal is to describe the steps that lead to the construction of the above final polynomial.

For that purpose we suggest that the reader forgets the above proof and assumes that $D_{3}^{10}$ is realizable. Under this assumption there exists a real $3 \times 10$ matrix $A$ of homogeneous coordinates for $D_{3}^{10}$ with

$$
A=\left(\begin{array}{cccccccccc}
1 & 0 & 0 & a & d & -g & j & -m & -p & s \\
0 & 1 & 0 & -b & e & h & -k & n & -q & t \\
0 & 0 & 1 & c & -f & i & -l & -o & r & u
\end{array}\right) .
$$

It can be read from Fig. 1 that all variables $a, b, \ldots, u$ have to be positive. For example, we have $[124]=c>0$ for the oriented area [124] of the triangle $1,2,4$. With the method in Section 3, it can be shown that the positivity of all 21 variables together with the following 10 inequalities forms a reduced system for $D_{3}^{10}$, i.e., $D_{3}^{10}$ is realizable if and only if the inequality system (1)-(10) has a solution within the positive real numbers. We called $D_{3}^{10}$ a simplicial nonDesargues chirotope because the mutations of $D_{3}^{10}$ (see Section 3 ) have the 
structure of the Desargues-configuration.

$$
\begin{aligned}
& {[123][145]=b f-c e>0,} \\
& {[123][185]=e o-f n>0,} \\
& {[123][160]=h u-i t>0,} \\
& {[123][240]=c s-a u>0,} \\
& {[123][256]=f g-d i>0,} \\
& {[123][296]=i p-g r>0,} \\
& {[123][364]=b g-a h>0,} \\
& {[123][374]=a k-b j>0,} \\
& {[123][350]=d t-e s>0,} \\
& {[123]^{2}[789]=r j n-\ln p-\operatorname{lm} q-o j q-o k p-r k m>0 .}
\end{aligned}
$$

To decide the realizability of $D_{3}^{10}$, we could proceed from this point on by naive variable elimination: consider for example the variable $c$ which is contained only in (1) and in (4). All variables being positive, these two inequalities can be rewritten as

$$
\frac{a u}{s}<c<\frac{b f}{e} .
$$

Hence, a real number $c$ satisfying (1) and (4) exists if and only if

$$
b f s-a e u>0 \text {, }
$$

and the system (1)-(10) can be replaced by the nine inequalities (2), (3), (5), (6), (7), (8), (9), (10), and (11) in one less variable.

Such elementary solving techniques can be used finally to derive $0<0$ which shows the contradiction. In order to obtain a final polynomial from this derivation, we trace the steps which lead to new polynomials as in (11) by forming positive linear combinations of vanishing polynomials. Therefore, we consider all inequalities as (vanishing) Grassmann-Plücker identities under the additional condition that all occurring brackets are positive.

$$
\begin{aligned}
p_{1} & =[123][145]-b f+c e, \\
p_{2} & =[123][185]-e o+f n, \\
p_{3} & =[123][160]-h u+i t, \\
p_{4} & =[123][240]-c s+a u, \\
p_{5} & =[123][256]-f g+d i, \\
p_{6} & =[123][296]-i p+g r, \\
p_{7} & =[123][364]-b g+a h, \\
p_{8} & =[123][374]-a k+b j, \\
p_{9} & =[123][350]-d t+e s, \\
p_{10} & =[123][123][789]-r j n+\ln p+\operatorname{lm} q+o j q+o k p+r k m .
\end{aligned}
$$


Solving for $c$ as in the derivation of (11) yields the new polynomial

$$
p_{11}:=s p_{1}+e p_{4}=[123][145] s+[123][240] e-b f s+a u e .
$$

Solving for $h$ yields

$$
p_{12}:=a p_{3}+u p_{7}=[123][160] a+[123][364] u-b g u+i t a .
$$

Solving for $d$ yields

$$
p_{13}:=t p_{5}+i p_{9}=[123][256] t+[123][350] i+e i s-f g t .
$$

Solving for $t$ yields

$$
\begin{aligned}
p_{14}:= & f g p_{12}+i a p_{13} \\
= & {[123][160] a f g+[123][364] f g u+[123][256] \text { ait } } \\
& +[123][350] a i i+\text { aeiis }- \text { bfggu. }
\end{aligned}
$$

Solving for $s$ yields

$$
\begin{aligned}
p_{15}:= & \text { aeiip }_{11}+b f p_{14} \\
= & {[123][145] \text { aeiis }+[123][240] \text { aeeii }+[123][160] a b f f g+[123][364] b f f g u } \\
& +[123][256] \text { abfit }+[123][350] a b f i i+u(a e i-b f g)(a e i+b f g)
\end{aligned}
$$

Partially solving for $j$ yields

$$
\begin{aligned}
p_{16}:= & r n p_{8}+b p_{10} \\
= & {[123][374] r n+[123][123][789] b } \\
& -a k r n+b \ln p+b l m q+b o j p+b o k p+b r k m .
\end{aligned}
$$

Partially solving for $r$ yields

$$
\begin{aligned}
p_{17}:= & g p_{16}+a k n p_{6} \\
= & {[123][296] a k n+[123][374] g r n+[123][123][789] b g } \\
& -a i k n p+b g l n p+b g l m q+b g j o p+b g k p o+b g r k m .
\end{aligned}
$$

Partially solving for $o$ yields

$$
\begin{aligned}
p_{18}:= & b g k p p_{2}+e p_{17} \\
= & {[123][296] a e k n+[123][374] \text { egrn }+[123][123][789] \text { beg }+[123][185] \text { bgkp } } \\
& +b e g(\operatorname{lnp}+\operatorname{lm} q+j o p+r k m)-k n p(a e i-b f g) .
\end{aligned}
$$


Finally, solving for $(a e i-b f g)$ yields

$$
\begin{aligned}
p_{19}:= & u(a e i+b f g) p_{18}+k n p p_{15} \\
= & {[123][145] \text { aeiiknps }+[123][240] \text { aeeiiknp }+[123][160] a b f f g k n p } \\
& +[123][364] b f f g k n p u+[123][256] \text { abfiknpt }+[123][350] a b f i k n p \\
& +[123][185] b g k p u(a e i+b f g)+[123][296] a e k n u(a e i+b f g) \\
& +[123][374] \text { egrnu }(a e i+b f g) \\
& +([123][123][789]+\operatorname{lnp}+\operatorname{lmq}+\text { jop }+r k m) \text { begu }(a e i+b f g) .
\end{aligned}
$$

To see that $p_{19}$ equals the above final polynomial, we would have to replace all variables by brackets according to the matrix $A$, e.g., $a$ by $[234], b$ by $[134], \ldots$, etc. With these substitutions, the expressions $p_{1}, p_{2}, \ldots, p_{10}$ equal the syzygy coefficients in the first representation of the final polynomial, e.g., $p_{1}=\{1 \mid 2345\}$, $p_{2}=-\{2 \mid 1340\}$, which completes the argument.

\section{Inequality Reduction for Chirotopes}

We have seen in the example in Section 2 that simplifying the original problem to a relatively small inequality system, which still carries the entire information, is a very helpful first step in deciding the realizability of geometric structures. In this section we describe a method of constructing a small reduced system for a given simplicial $d$-chirotope $\chi$ with $n$ points. Here $\mathscr{R} \subset \Lambda(n, d)$ is called a reduced system for $\chi$ if $\chi$ is uniquely determined by its restriction to $\mathscr{R}$, i.e., $\left.\chi^{\prime}\right|_{\mathscr{R}}=\left.\chi\right|_{\mathscr{R}}$ implies $\chi^{\prime}=\chi$ for every chirotope $\chi^{\prime}$.

As before, we use the abbreviation $\{\sigma \mid \tau\}:=\left\{\sigma_{1} \cdots \sigma_{d-2} \mid \tau_{1} \cdots \tau_{4}\right\}$ for the three term syzygies

$$
\begin{array}{r}
{\left[\sigma_{1} \cdots \sigma_{d-2} \tau_{1} \tau_{2}\right] \cdot\left[\sigma_{1} \cdots \sigma_{d-2} \tau_{3} \tau_{4}\right]} \\
-\left[\sigma_{1} \cdots \sigma_{d-2} \tau_{1} \tau_{3}\right] \cdot\left[\sigma_{1} \cdots \sigma_{d-2} \tau_{2} \tau_{4}\right] \\
+\left[\sigma_{1} \cdots \sigma_{d-2} \tau_{1} \tau_{4}\right] \cdot\left[\sigma_{1} \cdots \sigma_{d-2} \tau_{2} \tau_{3}\right]
\end{array}
$$

where $\tau \in \Lambda(n, 4), \sigma \in \Lambda(n, d-2)$. A syzygy $\{\sigma \mid \tau\}$ is said to determine a tuple $\left[\sigma \tau_{i} \tau_{j}\right]$ in $\chi$ if $\chi\left(\sigma, \tau_{i}, \tau_{j}\right)$ is uniquely determined by the values of $\chi$ for the other five tuples occurring in $\{\sigma \mid \tau\}$ and the chirotope condition for that syzygy. Given a subset $\mathscr{D} \subset \Lambda(n, d)$, then $(\mathscr{D}\rangle$ denotes the closure of $\mathscr{D}$ in the following sense: we add to $\mathscr{D}$ the set of brackets $\left[\sigma, \tau_{i}, \tau_{j}\right] \in \Lambda(n, d)$ which are determined in $\chi$ by some syzygy $\{\sigma \mid \tau\}$ whose other five brackets are already in $\mathscr{D}$. By iterating this process we eventually get $\langle\mathscr{D}\rangle$.

The problem is now to find a small subset $\mathscr{D} \subset \Lambda(n, d)$ with $\langle\mathscr{D}\rangle=\Lambda(n, d)$. In this section we describe an algorithm for constructing such a reduced system. 

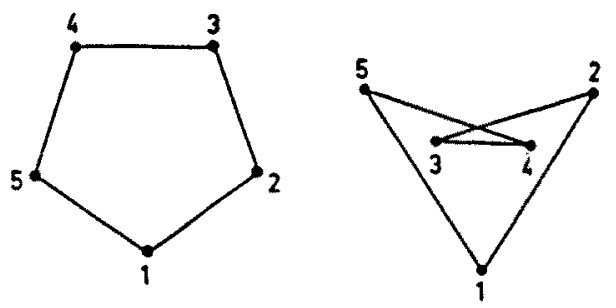

Fig. 2. The chirotopes $\chi_{5}$ and $\chi$ agree for all mutations of $\chi_{5}$.

We first consider $d$-tuples $\lambda$ which are necessarily contained in every reduced system of $\chi$ : we say that $\lambda \in \Lambda(n, d)$ is a mutation if it is not determined by any three-term syzygy [24], and we write $\operatorname{Mut}(\chi)$ for the set of mutations of $\chi$. Because of the results of [24], the mutations of a simplicial $d$-chirotope $\chi$ are in one-to-one correspondence to the simplicial regions of the associated arrangement of pseudohyperplanes, and $|\operatorname{Mut}(\chi)| \geq n$ if $\chi$ is realizable and $n$ is the number of points of $\chi$.

There are chirotopes $\chi$ for which $\operatorname{Mut}(\chi)$ is already a reduced system, e.g., the chirotope $R_{3}^{9}$ to be discussed in Section 5 has this property. In general, this cannot be expected. The alternating chirotope $\chi_{5}: \Lambda(5,3) \rightarrow\{+1\}$ has mutations $\operatorname{Mut}(\chi):=\{[123],[234],[345],[145],[125]\}$ but there is another chirotope $\chi: \Lambda(5,3) \rightarrow\{-1,+1\}$ with

$$
\chi(1,2,3)=\chi(2,3,4)=\chi(3,4,5)=\chi(1,4,5)=\chi(1,2,5)=+1
$$

see Fig. 2.

Since all later computations are most conveniently carried out with respect to a basis $\beta \in \Lambda(n, d)$, it is reasonable to add the set

$$
V_{\beta}:=\left\{\left[\beta_{1}, \ldots, \beta_{i-1}, k, \beta_{i+1}, \ldots, \beta_{d}\right] \mid k \notin \beta, i \in\{1, \ldots, d\}\right\}
$$

of "variables" (with respect to $\beta$ ) to the set of mutations. Observe that we are free to choose among $\left(\begin{array}{l}n \\ d\end{array}\right)$ possible bases.

Remark 3.1. Given any $\lambda \in \Lambda(n, d)$, the expansion of $[\lambda]$ with respect to a basis $\beta$ reads

$$
[\lambda]=\operatorname{det}\left(\begin{array}{cccc}
{\left[\lambda_{1} \beta_{2} \cdots \beta_{d}\right]} & {\left[\beta_{1} \lambda_{1} \cdots \beta_{d}\right]} & \cdots & {\left[\beta_{1} \beta_{2} \cdots \lambda_{1}\right]} \\
{\left[\lambda_{2} \beta_{2} \cdots \beta_{d}\right]} & {\left[\beta_{1} \lambda_{2} \cdots \beta_{d}\right]} & \cdots & {\left[\beta_{1} \beta_{2} \cdots \lambda_{2}\right]} \\
\vdots & \vdots & \ddots & \vdots \\
{\left[\lambda_{d} \beta_{2} \cdots \beta_{d}\right]} & {\left[\beta_{1} \lambda_{d} \cdots \beta_{d}\right]} & \cdots & {\left[\beta_{1} \beta_{2} \cdots \lambda_{d}\right]}
\end{array}\right)
$$

where without loss of generality $[\beta]=1$ is assumed. 
The above $d \times d$ determinant reduces to a $k \times k$ determinant where $k=|\lambda \backslash \beta|$. In order to choose a suitable basis $\beta$, we introduce a certain monotone "weight" function $w: N \rightarrow N$. Let $\beta \in \Lambda(n, d)$ such that the expression $v(\beta):=$ $\sum_{\lambda \in \operatorname{Mut}(\chi)} w(|\lambda \backslash \beta|)$ is minimized. In practice the following two weight functions turned out to be most useful:

(i) Counting the number of "too big" determinants:

$$
w(k)= \begin{cases}0 & \text { if } k \leq m \\ 1 & \text { if } k>m\end{cases}
$$

where $m$ is fixed.

(ii) Counting the total number of occurrences of variables in determinants: $w(k):=k^{2}$.

Now the following "filling up" algorithm determines a small reduced system.

\section{Algorithm 3.2.}

Input: Simplicial chirotope $\chi: \Lambda(n, d) \rightarrow\{-1,+1\}, \beta \in \Lambda(n, d)$.

Output: Small reduced system $\mathscr{R}$ for $\chi$.

1. Let $\mathscr{R}:=\operatorname{Mut}(\chi) \cup V_{\beta}, \mathscr{M}:=\varnothing$.

2. Determine $\mathscr{D}:=\langle\mathscr{R}\rangle$.

3. If $\mathscr{D}=\Lambda(n, d)$ :

3.1. Then GO TO 5 .

3.2. Else: Pick $\mu \in \Lambda(n, d) \backslash \mathscr{D}$ such that $|\mu \backslash \beta|$ is minimal.

4. Let $\mathscr{R}:=\mathscr{R} \cup\{\mu\}, M:=\mathscr{M} \cup\{\mu\}$. Go to 2 .

5. If $\mathscr{M}:=\varnothing$.

5.1. Then STOP, $\mathscr{R}$ is a reduced system.

5.2. Else: Pick $\lambda \in \mathscr{M}, \mathscr{M}:=\mathscr{M} \backslash\{\lambda\}$.

5.3. If $\langle\mathscr{R} \backslash\{\lambda\}\rangle=\Lambda(n, d)$, then $\mathscr{R}=\mathscr{R} \backslash\{\lambda\}$.

5.4. GO TO 5 .

At this point we still have the freedom to switch to another basis $\beta$ in order to get a more simplified reduced system.

\section{On the Construction of Final Polynomials}

Once a reduced system for an oriented matroid is constructed, we are left with the problem of deciding whether this system has a solution within the real numbers. In principle this decision can be made with Collins's algebraic cylindrical decomposition method [14] or any other decision procedure for real algebraic varieties. A new algorithm with a better asymptotic complexity than Collins's method has recently been obtained by Grigoryev and Vorobjov [17].

In practice, however, there is still a long way to go before these general purpose methods can be successfully applied in solving problems relevant to research 
in geometry. As an illustration consider the problem of finding a symmetric embedding of Möbius's torus with seven vertices in dimension 3 which has been studied in [6]. This simplest of all cases that we were interested in could be reduced by the methods discussed in Section 3 to the following seven inequalities in four variables:

$$
\begin{aligned}
& d>0, \quad b-1>0, \quad-c>0, \quad a-b+a c>0, \\
& b-a d>0, \quad a d+b c-b d>0, \quad b+c+d-a d-1>0 .
\end{aligned}
$$

B. Kutzler solved this system with the SAC-II implementation of Collins's method at the University of Linz in about 2 hours of CPU time, and slightly bigger systems could not be solved within 24 CPU hours. Nevertheless, there is some hope that Collins's algorithm can be significantly improved for our special type of problems, and we expect better computational results in the near future.

In the following we summarize some underlying and guiding ideas which, in all problems studied so far, have lead to final polynomial proofs for the nonrealizability of the oriented matroids in question.

While the existence of such a final polynomial in the case of nonrealizability is a consequence of a real version of Hilbert's Nullstellensatz [27], it is still very hard in practice to find the final polynomial. Substantial experience has been gained in calculating by hand with the support of computer-aided reductions. Yet our approach is still far from being complete and generally applicable.

So far final polynomials have been applied in different geometrical problems of interest such as the smallest nonrealizable matroid polytope $M_{963}^{9}$ [1], [2], in the case of Altshuler's sphere $M_{425}^{10}$, [7], in the two examples given in this paper, and in searching for symmetrical realizations of a manifold with a minimal number of vertices [5].

In all these cases the reduced system was small enough, and the solving technique used to find a solvability sequence or used in the above example to get inequality (11), etc., succeeded and was afterwards transformed as above into a suitable polynomial in the syzygy ideal to get a final polynomial.

Perhaps it was the special structure of these examples, they were homogeneous in nature, they had integer coefficients, and at the beginning all variables occurred linearly. A careful check was needed to discover how to start solving for variables; this led to hand-calculated decisions in all these cases.

Computer-aided implementations using these properties and/or a special variant of Collins's method might bring a substantial improvement in this direction. For a new computational approach see [8].

\section{A Configuration Without a Solvability Sequence}

In [10] the first and the third author introduced the concept of solvability sequences as a sufficient algorithmic criterion for the realizability of simplicial chirotopes. This criterion has been used to decide geometric realizability in a 
large number of instances [11]. It is an important property of chirotopes with a solvability sequence that their realization space is contractible [10, Theorem 5$]$.

Although an affirmative answer to this question was extremely unlikely, it remained an open problem whether all realizable chirotopes do have a solvability sequence, in which case nonrealizability of simplicial chirotopes could have been proven in an exhaustive search of all variable orderings. In this section we disprove this conjecture by giving an example, discovered by the second author, of a simplicial 3-chirotope with nine vertices which does not admit a solvability sequence.

Recall the following definitions from Section 5 of [10]. Let $\chi$ be a simplicial $d$-chirotope with $n$ elements. Pick a basis $\beta \in \Lambda(n, d)$, and, as in Section 3 , let $V_{\beta}$ denote the set of (bracket) variables with respect to this basis. Viewing the $\Delta \in \Lambda(n, d)$ as coordinate functions of the realization space $O_{\chi}$ of $\chi$, every $\Delta \epsilon$ $\Lambda(n, d)$ can be expressed as a determinant in certain variables from $V_{\beta}$ using Remark 3.1.

For a given total order $\left(v_{1}, \ldots, v_{d(n-d)}\right)$ of the set of variables $V_{\beta}$ define

$$
\Lambda_{i}:=\left\{\Delta \in \Lambda(n, d): \partial \Delta / \partial v_{j}=0 \text { for } j>i\right\}
$$

The sequence $\left(v_{1}, \ldots, v_{d(n-d)}\right)$ is called a solvability sequence for $\chi$ if the following condition holds:

for all $\eta_{1}, \ldots, \eta_{i-1} \in R$ with

$$
\operatorname{sign} \Delta\left(\eta_{1}, \ldots, \eta_{i-1}\right)=\chi(\Delta) \quad \text { for all } \Delta \in \Lambda_{i-1}
$$

there exists an $\eta_{i} \in R$ such that

$$
\operatorname{sign} \Delta\left(\eta_{1}, \ldots, \eta_{i}\right)=\chi(\Delta) \quad \text { for all } \Delta \in \Lambda_{i}
$$

In other words, $\left(v_{1}, \ldots, v_{d(n-d)}\right)$ is a solvability sequence for $\chi$ if the "greedy method" of assigning real numbers $\eta_{i}$ to the variables $v_{i}$ in the prescribed order necessarily yields a coordinatization of $\chi$.

Naturally, a similar definition makes sense on the level of points. We call a $d$-chirotope $\chi$ on an $n$-element set $E$ reducible if either $|E|=d$, that is, $\chi$ is a simplex, or there is a point $e \in E$ such that for all $X \subset R^{d}$ with $X \in \sigma_{\chi \backslash \text { (e\} }}$ there exists an $x \in R^{d}$ such that $X \cup x \in O_{X}$. Notice that every 2-chirotope is reducible. The motivation for this definition is that it suffices to consider only nonreducible cases in order to decide realizability. Consider the 3-chirotope $R_{3}^{9}$ associated with the point configuration in Fig. 3.

Theorem 5.1. The realizable chirotope $R_{3}^{9}$ is not reducible.

Proof. Since $R_{3}^{9}$ has the combinatorial symmetry $\sigma=(123456)(789)$ it is sufficient to consider representatives from the two orbits of $\sigma$, say, point 1 and point 8 . In 


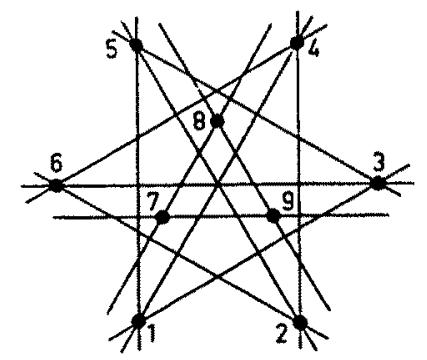

Fig. 3. The simplicial chirotope $R_{3}^{9}$ without a solvability sequence.

Fig. 4(a) and (b) realizations of $R_{3}^{9} \backslash 1$ and $R_{3}^{9} \backslash 8$ are given, which cannot be extended to a realization of $R_{3}^{9}$. To see this, consider the thick lines in Fig. 4(a) and (b). It is easy to check that there is no point simultaneously satisfying all orientation conditions as indicated by the arrows at the thick lines. Hence, by definition $R_{3}^{9}$ is not reducible.

We remark that the line arrangement associated with the oriented matroid $R_{3}^{9}$ can be obtained by deletion from the unique tight arrangement with 10 lines, see Figure 5 of [23], which also is not reducible. An oriented matroid $\chi$ being tight means that every mutation of a rank-2 minor of $\chi$ induces a mutation of $\chi$.

Proposition 5.2. If a simplicial 3-chirotope $\chi$ admits a solvability sequence then $\chi$ is reducible.

Proof. Let $\chi$ be a simplicial 3-chirotope with $n$ points, and assume that for some basis $\beta \in \Lambda(n, 3)$ the set of variables $V_{\beta}$ can be ordered to give a solvability sequence for $\chi$. Suppose that $v_{3(n-3)}:=\left(\beta \backslash \beta_{i}\right) \cup k, k \notin \beta$, is the last element of this solvability sequence. As above, we write

$$
\Lambda_{3(n-3)-1}:=\left\{\Delta \in \Lambda(n, d): \frac{\partial \Delta}{\partial v_{3(n-3)}}=0\right\} .
$$

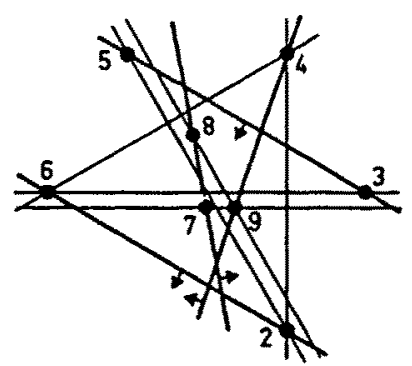

(a)

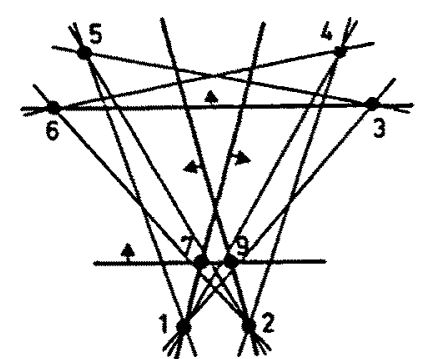

(b)

Fig. 4. $R_{3}^{9}$ is not reducible. 
To see that $\chi$ is reducible, we need to show that every realization $X \subset R^{3}$ of $\chi \backslash k$ extends to a realization $X \cup x_{k} \subset R^{3}$ of $\chi$. Every rank-2 oriented matroid being reducible, the induced realization of $\left(\chi / \beta_{i}\right) \backslash k$ can be extended to a realization of $\left(\chi / \beta_{i}\right)$. This means that we can assign real numbers to the variables $\left[\left(\beta \backslash \beta_{j}\right) \cup k\right], j \in\{1,2,3\} \backslash\{i\}$, in a way that is compatible with $\chi$. This assignment together with $X$ gives us real numbers $\eta_{1}, \ldots, \eta_{3(n-3)-1}$ such that $\operatorname{sign}(\Delta)=\chi(\Delta)$ for all $\Delta \in \Lambda_{3(n-3)-1}$.

Since $v_{3(n-3)}$ was assumed to be the last element in a solvability sequence, we can find an $\eta_{3(n-3)} \in R$ which completes the sequence $\eta_{1}, \ldots, \eta_{3(n-3)-1}$ to a realization of $\chi$. Retranslated in geometric language: every realization of $\chi \backslash k$ extends to a realization of $\chi$. This proves the claim.

Theorem 5.1 together with Proposition 5.2 implies

Corollary 5.3. The realizable chirotope $R_{3}^{9}$ does not admit a solvability sequence.

Let us close this section with the remark that although $R_{3}^{9}$ does not admit a solvability sequence, it does fulfill the isotopy property. To see this, consider the following short nondeterministic construction algorithm for all realizations of $R_{3}^{9}$.

1. Realize $R_{3}^{9} \backslash\{1,6\}$. (Every 3 -chirotope with seven points is realizable.)

2. Delete point 7 .

3. Insert points 1 and 6.

4. Insert point 7 .

It can be shown that every realization of $R_{3}^{9}$ can be arrived at in this way, and, moreover, the induced maps on the realization spaces are homotopy equivalences, compare [22]. Hence, this construction procedure shows that the realization space of $R_{3}^{9}$ is contractible, therefore path-connected.

In Fig. 5(a)-(c) an example of this construction sequence is given. Details of the topological argument will be omitted here.

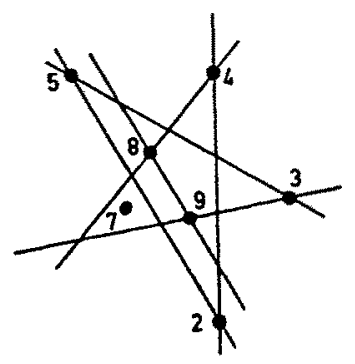

(a)

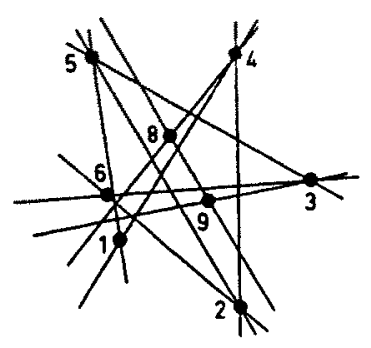

(b)

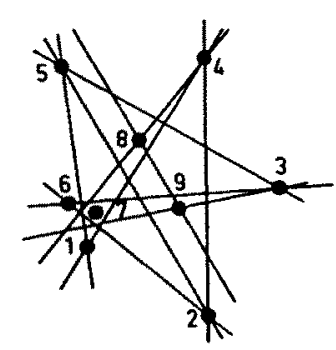

(c)

Fig. 5. Geometric construction showing the isotopy property for $R_{3}^{9}$. 


\section{Existence of Final Polynomials for Matroids and Oriented Matroids}

The main theme of this paper is the construction of final polynomials as nonrealizability proofs for oriented matroids. Naturally, it is a fundamental question whether this method is generally applicable, i.e., whether for every nonrealizable object there exists a final polynomial.

Using methods from real algebraic geometry, it can be shown that the answer to this question is "yes." A discussion of the algebraic details and proofs for all theorems in this section are contained in the monograph [27]. Here we restrict ourselves to giving the precise definition of final polynomials for matroids and oriented matroids and to stating, without proof, results of the existence for final polynomials. We also include a final polynomial proof for Pappus's theorem as an example for the unoriented case.

Given integers $n \geq d \geq 1$ and a field $K$, consider the polynomial algebra $K[\Lambda(n, d)]$ freely generated over $K$ by all brackets $[\lambda], \lambda \in \Lambda(n, d)$. As usual, we write $\left[\lambda_{\pi(1)} \cdots \lambda_{\pi(d)}\right]:=\operatorname{sign} \pi \cdot\left[\lambda_{1} \cdots \lambda_{d}\right]$ for any permutation $\pi$. Let $I_{n, d}^{K}$ denote the ideal generated in $K[\Lambda(n, d)]$ by all quadratic syzygies

$$
\sum_{i=1}^{d+1}(-1)^{i} \cdot\left[\lambda_{1}, \ldots, \lambda_{i-1}, \lambda_{i+1}, \ldots, \lambda_{d+1}\right] \cdot\left[\lambda_{i}, \mu_{1}, \ldots, \mu_{d-1}\right]
$$

where $\lambda \in \Lambda(n, d+1), \mu \in \Lambda(n, d-1)$. Viewing $K[\Lambda(n, d)]$ as the ring of polynomial functions on the $\left(\begin{array}{l}n \\ d\end{array}\right)$-dimensional vector space $\wedge_{d} K^{n}, I_{n, d}^{K}$ is the vanishing ideal of the Grassmann variety of simple $d$-vectors. The coordinate ring $K[\Lambda(n, d)] / I_{n, d}^{K}$ of this variety is (in the terminology of White [29]) the bracket ring with coefficients in $K$ of the uniform rank- $d$ matroid on an $n$-element set.

Now let $M$ be any rank- $d$ matroid on $E=\{1,2, \ldots, n\}$ [30]. We assign to $M$ the two sets of all bracket polynomials that must (resp. cannot) vanish under a coordinatization. Let $I_{M}^{K}$ denote the ideal in $K[\Lambda(n, d)]$ which is generated by $\{[\lambda], \lambda$ dependent in $M\}$, and let $S_{M}^{K}$ denote the multiplicative semigroup with unit generated by $\{[\lambda], \lambda$ is basis of $M\}$. In other words, $I_{M}^{K}$ consists of all linear combinations of nonbasis brackets with polynomial coefficients, and $S_{M}^{K}$ consists of all nonzero monomials which are products of basis brackets.

A polynomial $f \in K[\Lambda(n, d)]$ is called a final polynomial for $M$ if $f \in I_{n, d}^{K} \cap$ $\left(S_{M}^{K}+I_{M}^{K}\right)$. With this definition we can state the desired "theorem of the alternate" for realizability of matroids.

Theorem 6.1. Let $M$ be a matroid and let $K$ be a field. Then one and only one of the following statements is true:

(i) There exists a final polynomial for $M$ with coefficients in $K$.

(ii) $M$ is realizable over some finite algebraic field extension of $K$.

In particular, we have

Corollary 6.2. A matroid $M$ is not realizable over an algebraically closed field $K$ if and only if there exists a final polynomial for $M$ with coefficients in $K$. 


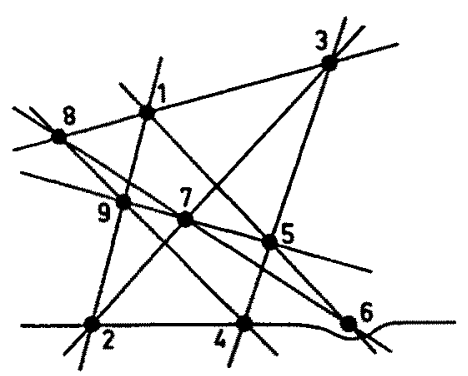

Fig. 6. The non-Pappus matroid NP.

Example 6.3. The non-Pappus matroid NP, see Fig. 6 , is the rank-3 matroid on $E=\{1,2, \ldots, 9\}$ defined by its nonbases (three point lines)

$$
\mathcal{N}=\{[129],[138],[156],[345],[489],[579],[678],[237]\}
$$

This matroid is not realizable over any field $K$. For, assume there exist $x_{i} \in K^{3}$, $i=1, \ldots, 9$, such that $x_{i}, x_{j}, x_{k}$ are linearly dependent if and only if $[i j k] \in \mathcal{N}$, then $x_{1}, x_{3}, x_{8}$ and $x_{5}, x_{7}, x_{9}$ being both dependent implies by Pappus's theorem that the three vectors $x_{2}=\left(x_{1} \vee x_{9}\right) \wedge\left(x_{3} \vee x_{7}\right), x_{4}=\left(x_{3} \vee x_{5}\right) \wedge\left(x_{8} \vee x_{9}\right)$, and $x_{6}=$ $\left(x_{1} \vee x_{5}\right) \wedge\left(x_{8} \vee x_{7}\right)$ are linearly dependent as well; in contradiction to [246] $\notin \mathcal{N}$.

Let us give a nonrealizability proof for NP (and hence a proof for Pappus's theorem) by establishing a final polynomial. The polynomial

$$
\begin{aligned}
p:= & \{4 \mid 1267\}[148][157][437][197]+\{1 \mid 2479\}[148][157][437][467] \\
& +\{1 \mid 3478\}[149][247][157][467]+\{1 \mid 4567\}[148][247][347][197] \\
& +\{4 \mid 1357\}[148][427][167][179]+\{4 \mid 1789\}[247][157][167][413] \\
& +\{7 \mid 1459\}[247][148][167][143]+\{7 \mid 1468\}[247][157][149][134] \\
& +\{7 \mid 1234\}[194][148][157][467]
\end{aligned}
$$

is a linear combination of three term syzygies and hence contained in the above defined ideal $I_{9,3}^{K}$. By expanding the expressions $\{i \mid j k l m\}$, we obtain a sum of 27 monomials of degree six, 18 of which vanish by cancelling in pairs. It remains

$$
\begin{aligned}
& p=[246][147][148][157][437][197]+[\underline{[129]}[147][148][157][437][467]
\end{aligned}
$$

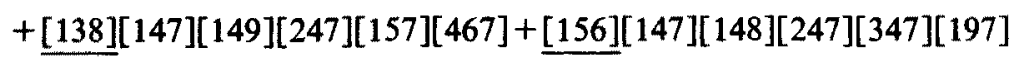

$$
\begin{aligned}
& -\underline{[435][147][148][427][167][179]-[489][147][247][157][167][413]} \\
& +[759][147][247][148][167][143]+[768][147][247][157][149][134] \\
& +[723][147][194][148][157][467] \text {. }
\end{aligned}
$$


Since all underlined brackets are contained in $\mathcal{N}$, the last eight summands are contained in the ideal $I_{N P}^{K}$. On the other hand, [246][147][148][157][437][197] $\epsilon$ $S_{N P}^{K}$, and hence $p \in I_{9,3}^{K} \cap\left(S_{N P}^{K}+I_{N P}^{K}\right)$, that is, $p$ is a final polynomial for the non-Pappus matroid $N P$.

In considering the above bracket ring and ideals with integer coefficients, we obtained the following generalization of this example.

Remark 6.4. A matroid $M$ is not realizable over any field $K$ if and only if there exists a final polynomial for $M$ with integer coefficients.

Let us now turn to the case of oriented matroids or chirotopes. Therefore, assume that $K$ is an ordered field, and let $K[\Lambda(n, d)]$ and $I_{n, d}^{K}$ be as above. Given a (not necessarily simplicial) $d$-chirotope $\chi$ on $E=\{1,2, \ldots, n\}$, we assign to $\chi$ the three sets $I_{\chi}^{K}, N_{\chi}^{K}$, and $P_{\chi}^{K}$ of bracket polynomials which are respectively zero, nonnegative, and positive in any realization of $\chi$. First let (as above) $I_{x}^{K}$ denote the ideal in $K[\Lambda(n, d)]$ which is generated by $\{[\lambda] \in \Lambda(n, d), \chi(\lambda)=0\}$. For simplicial chirotopes we have clearly $I_{x}^{K}=\{0\}$.

Let $P_{x}^{K}$ denote the multiplicative semigroup with unit generated by the positive brackets $\{[\lambda], \chi(\lambda)=+1\}$, the negated negative brackets $\{-[\lambda], \chi(\lambda)=-1\}$, and the positive elements in the ordered field $K$. Finally, define $N_{\chi}^{K}$ to be the quadratic semiring in $K[\Lambda(n, d)]$ which is generated by $P_{\chi}^{K}$ and the set $K[\Lambda(n, d)]^{2}$ of all squares.

A polynomial $f \in K[\Lambda(n, d)]$ is called a final polynomial for $\chi$ if $f \in I_{n, d}^{K} \cap$ $\left(I_{\chi}^{K}+N_{\chi}^{K}+P_{\chi}^{K}\right)$. (Compare with the example in Section 2.) With this definition we have

Theorem 6.5. Let $\chi$ be a chirotope and let $K$ be an ordered field. Then one and only one of the following statements is true:

(i) There exists a final polynomial for $M$ with coefficients in $K$.

(ii) $M$ is realizable over some finite algebraic ordered field extension of $K$.

The proof of Theorem 6.5 is derived from the real version of the Nullstellensatz using straightforward algebraic methods. See Section 4 of [27] for a detailed exposition. Since every realizable chirotope (over some ordered field) is realizable over the real algebraic numbers we have

Corollary 6.6. A chirotope $\chi$ is not realizable if and only if there exists a final polynomial for $\chi$ with rational coefficients.

\section{References}

1. Altshuler, A.; Bokowski, J.; Steinberg, L.: The classification of simplicial 3-spheres with nine vertices into polytopes and non-polytopes, Discrete Math. 31 (1980), 115-124.

2. Antonin, C.: Ein Algorithmusansatz für Realisierungsfragen im $E^{d}$ getestet an kombinatorischen 3-Sphären. Staatsexamensarbeit, Bochum, 1982. 
3. Bachem, A.: Convexity and optimization in discrete structures. In Convexity and Applications (ed. P. M. Gruber and J. Wills), Birkhäuser, Basel, 1983.

4. Bland, R.; Las Vergnas, M.: Orientability of matroids, J. Combin. Theory Ser. B 24 (1978), 94-123.

5. Bokowski, J.; Brehm, U.: A New Polyhedron of Genus 3 with 10 Vertices. Colloquia Mathematica Societatis János Bolyai, Siofok, 1985.

6. Bokowski, J.; Eggert, A.: All realizations of Möbius' torus with seven vertices. Preprint No. 1009. Technische Hochschule Darmstadt, 1987.

7. Bokowski, J.; Garms, K.: Altshuler's sphere $M_{425}^{10}$ is not polytopal, European J. Combin. 8 (1987), 227-229.

8. Bokowski, J.; Richter, J.: On the finding of final polynomials, Manuscript, 1988.

9. Bokowski, J.; Shemer, I.: Neighborly 6-polytopes with 10 vertices, Israel J. Math. 58 (1987), 103-124.

10. Bokowski, J.; Sturmfels, B.: On the coordinatization of oriented matroids, Discrete Comput. Geom. 1 (1986), 293-306.

11. Bokowski, J.; Sturmfels, B.: Polytopal and non-polytopal spheres-an algorithmic approach, Israel J. Math. 57 (1987), 121-124.

12. Bokowski, J.; Sturmfels, B.: An infinite family of minorminimal nonrealizable simplicial 3chirotopes, submitted for publication.

13. Canny, J. F.: Robot motion planning and real geometry. Proc. 28th Annual IEEE Conference of Foundations of Computer Science, Los Angeles, 1987.

14. Collins, C.: Quantifier Elimination for Real Closed Fields by Cyclindrical Algebraic Decomposition (Proc. 2nd GI Conf. on Automata and Formal Languages). Lecture Notes in Computer Science, Vol. 33, Springer-Verlag, Berlin, 1975, pp. 134-163.

15. Dress, A.: Chirotops and oriented matroids, Bayreuth. Math. Schr. 21 (1986), 14-68.

16. Gelfand, I. M.; Goresky, R. M.; MacPherson, R. D.; Serganova, V.: Combinatorial geometries, convex polyhedra and Schubert cells, Adv. in Math. 63 (1987), 301-316.

17. Grigoryev, D. Y.; Vorobjov, N. N.: Solving systems of polynomial inequalities in subexponential time, J. Symbolic Comput., to appear.

18. Jaggi, B.; Mani-Levitska, P.: A simple arrangement of lines without the isotopy property. Manuscript, University Bern, 1988.

19. Lawrence, J.: Oriented matroids and multiple ordered sets, Linear Algebra Appl. 48 (1982), 1-12.

20. Ljubić, D.; Roudneff, J.-P.; Sturmfels, B.: Arrangements of lines and pseudolines without adjacent triangles, J. Combin. Theory Ser. A, to appear.

21. Mandel, A.: Topology of oriented matroids. Ph.D. Thesis, University of Waterloo, Ontario, 1981.

22. Richter, J.; Sturmfels, B.: On the geometric construction of oriented matroids and convex polytopes, submitted for publication.

23. Roudneff, J.-P.: On the number of triangles in simple arrangements of pseudolines in the real projective plane, Discrete Math. 60 (1986), 243-251.

24. Roudneff, J.-P.; Sturmfels, B.: Simplicial cells in arrangements and mutations of oriented matroids, Geom. Dedicata 27 (1988), 153-170.

25. Sturmfels, B.: Boundary complexes of convex polytopes cannot be characterized locally, J. London Math. Soc. 35 (1987), 314-326.

26. Sturmfels, B.: On the decidability of diophantine problems in combinatorial geometry, Bull. Amer. Math. Soc. 17 (1987), 121-124.

27. Sturmfels, B.: Computational Synthetic Geometry. Lecture Notes in Mathematics, Vol. 1355, Springer-Verlag, Heidelberg, 1988.

28. Sturmfels, B.: Computational algebraic geometry of projective configurations. In Symbolic Computations in Geometry, IMA Preprint Series No. 389, University of Minnesota, 1988.

29. White, N.: The bracket ring of a combinatorial geometry, 1, Trans. Amer. Math. Soc. 202 (1975), $79-103$.

30. White, N. (ed.): Theory of Matroids, Cambridge University Press, Cambridge, 1986.

31. White, N.: A nonuniform matroid which violates the isotopy conjecture, Discrete Comput. Geom. 4 (1989), 1-2.

Received May 18, 1987, and in revised form May 31, 1988. 